\title{
Spatial distribution of soil-transmitted helminths, including Strongyloides stercoralis, among children in Zanzibar
}

\author{
Stefanie Knopp ${ }^{1}$, Khalfan A. Mohammed ${ }^{2}$, I. Simba Khamis², Ali F. Mgeni ${ }^{2, \dagger}$, J. Russell \\ Stothard ${ }^{3}$, David Rollinson ${ }^{3}$, Hanspeter Marti ${ }^{4}$, Jürg Utzinger ${ }^{1}$ \\ ${ }^{1}$ Department of Public Health and Epidemiology, Swiss Tropical Institute, P.O. Box, CH-4002 Basel, \\ Switzerland; ${ }^{2}$ Helminth Control Laboratory Unguja, Ministry of Health and Social Welfare of Zanzibar, \\ P.O. Box 236, Zanzibar, United Republic of Tanzania; ${ }^{3}$ Wolfson Wellcome Biomedical Laboratories, \\ Department of Zoology, Natural History Museum, Cromwell Road, London SW7 5BD, United Kingdom; \\ ${ }^{4}$ Department of Medical and Diagnostic Services, Swiss Tropical Institute, P.O. Box, CH-4002 Basel, \\ Switzerland
}

${ }^{\dagger}$ Deceased December 2007

\begin{abstract}
A programme periodically distributing anthelminthic drugs to school-aged children for the control of soiltransmitted helminthiasis was launched in Zanzibar in the early 1990s. We investigated the spatial distribution of soiltransmitted helminth infections, including Strongyloides stercoralis, in 336 children from six districts in Unguja, Zanzibar, in 2007. One stool sample per child was examined with the Kato-Katz, Koga agar plate and Baermann methods. The point prevalence of the different helminth infections was compared to the geological characteristics of the study sites. The observed prevalences for Trichuris trichiura, Ascaris lumbricoides, hookworm and S. stercoralis were $35.5 \%, 12.2 \%, 11.9 \%$ and $2.2 \%$, respectively, with considerable spatial heterogeneity. Whilst T. trichiura and hookworm infections were found in all six districts, no A. lumbricoides infections were recorded in the urban setting and only a low prevalence $(2.2 \%)$ was observed in the South district. S. stercoralis infections were found in four districts with the highest prevalence $(4.0 \%)$ in the West district. The prevalence of infection with any soil-transmitted helminth was highest in the North A district (69.6\%) and lowest in the urban setting $(22.4 \%)$. A. lumbricoides, hookworm and, with the exception of the North B district, S. stercoralis infections were observed to be more prevalent in the settings north of Zanzibar Town, which are characterized by alluvial clayey soils, moist forest regions and a higher precipitation. After a decade of large-scale administration of anthelminthic drugs, the prevalence of soil-transmitted helminth infections across Unguja is still considerable. Hence, additional measures, such as improving access to adequate sanitation and clean water and continued health education, are warranted to successfully control soil-transmitted helminthiasis in Zanzibar.
\end{abstract}

Keywords: soil-transmitted helminths, Strongyloides stercoralis, spatial distribution, soil type, Zanzibar.

\section{Introduction}

Unguja and Pemba, the two islands belonging to the Zanzibar archipelago offshore Tanzania in East

Corresponding author:

Jürg Utzinger

Department of Public Health and Epidemiology

Swiss Tropical Institute, P.O. Box, CH-4002 Basel, Switzerland

Tel. +41 61284 8129; Fax +41612848105

E-mail: juerg.utzinger@unibas.ch
Africa, are highly endemic for worm infections (Albonico et al., 1997; Mohammed et al., 2008; Stothard et al., 2008). Indeed, filaria (Wuchereria bancrofti), schistosomes (Schistosoma haematobi$u m$ ) and the common soil-transmitted helminths (i.e. Ascaris lumbricoides, hookworm and Trichuris trichiura) co-exist and multiple infections of different helminth species are common (Mohammed et al., 2008; Rudge et al., 2008). Additionally, Strongyloides stercoralis, the most 
neglected of the soil-transmitted helminths (Bethony et al., 2006; Steinmann et al., 2007), is also endemic (Marti et al., 1996; Stoltzfus et al., 1997; Knopp et al., 2008).

The tropical climate of the Zanzibar archipelago and the poor hygienic conditions under which the socio-economically deprived rural dwellers live facilitate the development and transmission of these helminth infections. Whilst lymphatic filariasis and soil-transmitted helminthiasis are endemic across Unguja (Mohammed et al., 2008), urinary schistosomiasis is geographically restricted. The focality of urinary schistosomiasis is governed by the distribution of its intermediate host, i.e. Bulinus globosus (Stothard and Rollinson, 1997). This snail species, and hence urinary schistosomiasis, is absent in the south of the island (Stothard et al., 2002). New research has revealed that soil-transmitted helminth infections also show considerable spatial heterogeneity; indeed an elevated prevalence of A. lumbricoides and $T$. trichiura has been reported in the northern parts of Unguja (Stothard et al., 2008). One possible explanation is that the eggs of these soil-transmitted helminths remain infective for a long period of time in the alluvium and clayey sands that are the predominant soil type in North Unguja (Stothard et al., 2008). Clayey sands can be considered as a composite matrix of coarse and fine grains where the interaction between coarser and finer grain matrices affects the overall stress-strain behaviour of the soil (Monkul and Ozden, 2007). For the transmission of hookworm, a poverty-related lifestyle in combination with environmental factors such as a sandy soil type with a high moisture content, a suitable temperature, rainfall and sun exposure are key factors (Brooker et al., 2004; Saathoff et al., 2005b; Hotez, 2008). The ecological and epidemiological features that might explain the spatial heterogeneity of hookworm and S. stercoralis distribution in Unguja, however, remain to be investigated.

Due to the high prevalence of $S$. haematobium and soil-transmitted helminth infections in Zanzibar and their negative health impacts, especially among children, a national control programme was initiat- ed by the Zanzibar Ministry of Health in the early 1990s (Renganathan et al., 1995). Since 1994, single-dose oral praziquantel $(40 \mathrm{mg} / \mathrm{kg})$ against schistosomiasis, and single-dose oral mebendazole (500 $\mathrm{mg}$ ) against soil-transmitted helminthiasis, were administered, mainly through the existing education sector (Mohammed et al., 2008). From 2003 onwards, mebendazole has been replaced by singledose oral albendazole (400 mg) (Stothard et al., 2008). In 2001, a programme to eliminate lymphatic filariasis was established, distributing once yearly single-dose oral albendazole (400 mg) plus ivermectin $(200 \mu \mathrm{g} / \mathrm{kg})$ to the whole eligible population of Unguja (Mohammed et al., 2006). Ivermectin is not only effective against filaria and A. lumbricoides, but is also the drug of choice to treat $S$. stercoralis infections (Gann et al., 1994), and hence is likely to have an ancillary effect against strongyloidiasis.

Despite S. stercoralis being endemic in Unguja and Pemba (Marti et al., 1996; Stoltzfus et al., 1997), only one of the recently published articles discussing different aspects of soil-transmitted helminthiasis focussed on $S$. stercoralis (Knopp et al., 2008). The aim of the present study was to assess the prevalence and intensity of infection of soil-transmitted helminth infections, placing particular emphasis on S. stercoralis, among children from selected "madrassas" (Koran-schools) and primary schools in the six districts of Unguja. Finally, the spatial distribution of soil-transmitted helminth infections was considered in relation to known geological features.

\section{Materials and methods}

\section{Study area}

This study was carried out in Unguja, the main island of Zanzibar, in June 2007. Unguja has two annual wet seasons: the "Masika rains" from the south, lasting usually from mid-March to mid-June, and the "Vuli rains" from north-east, occurring during November and December. The average annual 
rainfalls for north Unguja are $1,800 \mathrm{~mm}$ and for South Unguja 1,500 mm (MDG-Centre, 2007). The average annual temperature in Unguja is $27^{\circ} \mathrm{C}$. The pedeology of Unguja soils range from alluvium to clayey sands with subordinate limestone (Kent et al., 1971). In the north-east of Unguja various soil-types constitute the ground whereas, at the western coastline and in the south, coral limestone areas are predominant (Calton et al., 1955; Hettige, 1990).

\section{Study population}

Stool samples were obtained from children visiting the "madrassas" of five villages, namely BandaMaji, Mahonda, Kitumba, Dole and Pete. These villages are situated in the North A, North B, Central, West and South districts, respectively, and were geographically localized using Google ${ }^{\mathrm{TM}}$-Earth 2008. All villages are quite remote, have a similar socioeconomic status with houses usually built with clayey walls and thatched coconut leafs. The majority of houses have no access to the power grid.

Additionally, stool samples were collected from children visiting five peri-urban primary schools (i.e. Nyerere, Rahaleo, Fuoni, Mwenge and Regeza Mwendo) belonging to the Urban district around Zanzibar Town. All surveyed villages were located between 5 and $40 \mathrm{~km}$ from Zanzibar Town.

\section{Field and laboratory procedures}

One day before visiting the Koran-schools, the respective "shehas" (heads) of the communities were informed about the purpose and procedures of the study and asked for permission. Stool containers, a marker-pen and a pre-numbered form to fill in the name, sex and age of the children attending school were given to the teachers who were instructed to ask each child, attending the afternoon class, to write down his/her name on the pre-numbered list and to record his/her unique identification number on the designated stool collection container. The containers were handed out to the children and they were invited to return the containers with a lime- sized sample of their next morning stool.

To minimize the risk of sample mix-up, a member of the Helminth Control Laboratory Unguja (HCLU) visited the Koran-schools early in the morning and called each child by name and compared the number on their filled container with the number on the list. The stool samples were transferred to the HCLU in Mianzini, Zanzibar Town. A similar stool collection procedure was implemented in the five urban primary schools, readily integrated in the so-called 24-school-survey, as part of an ongoing study on the epidemiology and control of soil-transmitted helminthiasis and urinary schistosomiasis (French et al., 2007).

Once the stool samples reached HCLU, they were promptly processed and examined by an experienced laboratory technician. In brief, stool samples of sufficient quantity were examined with three different methods according to the following priorities. First, a Kato-Katz thick smear was prepared using $41.7 \mathrm{mg}$ templates (Katz et al., 1972). Second, a groundnut-sized portion of each stool sample $(\sim 1 \mathrm{~g})$ was subjected to the Koga agar plate method (Koga et al., 1990). Third and finally, the Baermann technique was performed (García and Bruckner, 2001). Further details pertaining to these three methods for helminth diagnosis have been presented elsewhere (Steinmann et al., 2007; Knopp et al., 2008).

Kato-Katz thick smears were examined quantitatively, i.e. the number of eggs for each helminth species was counted and recorded separately. The Koga agar plate was used to determine the presence, or absence, of larvae of S. stercoralis and hookworm. The Baermann method was used for diagnosis of $S$. stercoralis larvae. For quality control purposes, random samples amounting to $5 \%$ of the Kato-Katz thick smears, were re-examined by a senior laboratory technician.

\section{Data management and analysis}

Data were double-entered in Microsoft Excel version 10.0 and checked for consistency using EpiData version 3.1 (EpiData Association, Odense, 
Table 1. Thresholds issued by the WHO for distinguishing light, moderate and heavy infection intensities (Montresor et al., 1998).

\begin{tabular}{llcr}
\hline Infection & \multicolumn{2}{c}{ Thresholds for each soil-transmitted helminth $\left(\mathrm{EPG}^{\mathrm{a}}\right)$} \\
\cline { 2 - 4 } intensity & A. lumbricoides & T. trichiura & Hookworm \\
\hline Light & $1-4,999$ & $1-999$ & $1-1,999$ \\
Moderate & $5,000-49,999$ & $1,000-9,999$ & $2,000-3,999$ \\
Heavy & $\geq 50,000$ & $\geq 10,000$ & $\geq 4,000$ \\
\hline
\end{tabular}

${ }^{\mathrm{a}} \mathrm{EPG}=$ eggs per gram of stool

Denmark). Statistical analyses were made with STATA version 9.2 (StataCorp., College Station, USA). The number of eggs per gram of stool (EPG) was obtained by multiplying the species-specific egg-count in a single Kato-Katz thick smear by 24 . We used thresholds issued by the World Health Organization (WHO) for distinguishing light, moderate and heavy infection intensities (Montresor et al., 1998), as showed in Table 1.

The geometric mean EPG was calculated for the children in the respective settings, using the following formula: $\exp \left(\sum \log (\mathrm{EPG}+1) / \mathrm{n}\right)-1$.

\section{Ethical considerations and treatment}

One part of this study was embedded in the island-wide parasitological surveys that are carried out in Unguja by the HCLU as part of their routine activities. Moreover, two schools were part of the 24-school survey that have been conducted once every year, starting in 2004, as a collaborative activity between the HCLU and the Natural History Museum (London, UK).

The institutional research commission of the Swiss Tropical Institute (Basel, Switzerland) and the institutional review board of the National Health Service Local Research Ethics Committee (application 03.36) of St. Mary's Hospital (London, UK), on behalf of the Natural History Museum/Imperial College, approved the study. The WHO (Geneva, Switzerland), and the Ministry of Health and Social Welfare (Stone Town, Zanzibar) cleared the study protocol.

Detailed information about the study was provid- ed to the "shehas" of the villages, the teachers of the Koran-schools and the headmasters of the urban primary schools. A member of the HCLU explained the study to the children. Written informed consent to all anticipated medical interventions, including parasitological surveys done at schools, was given by parents and/or legal guardians upon enrolment of their children at school. At the end of the study, children were treated regardless of their infection status with a single oral dose of albendazole $(400$ $\mathrm{mg}$ ). Those children found positive for $S$. stercoralis were also treated with a single oral dose of ivermectin $(200 \mu \mathrm{g} / \mathrm{kg})$.

\section{Results}

\section{Study compliance}

In total, 336 children from the six districts of Unguja submitted one stool sample each. The samples were subjected to three different methods for helminth diagnosis. Age and/or sex were not available for all children; sex was recorded for 309 $(92.0 \%)$ children out of which $51.8 \%$ were girls and $48.2 \%$ were boys, and age was recorded from 244 $(72.6 \%)$ children. The median age was 11 years with a range from 3 to 19 years. Hookworm diagnosis was performed on 334 (99.4\%) stool samples examined either with the Kato-Katz, or the Koga agar plate, or both methods. Results for A. lumbricoides and T. trichiura were available for 327 $(97.3 \%)$ children who had their stool samples examined with the Kato-Katz method. Finally, 319 $(94.9 \%)$ stool samples were subjected both to the 
Baermann and the Koga agar plate method for diagnosis of $S$. stercoralis.

\section{Helminth infections}

Table 2 shows that helminth infections are still prevalent among children in Unguja. With regard to the three common soil-transmitted helminths, A. lumbricoides was the only species that was absent in one of the surveyed settings, namely in the Urban district. $S$. stercoralis was the least common helminth infection. It was found in four of the six districts with an overall prevalence of $2.2 \%$ according to combined results from the Koga agar plate and the Baermann methods. The highest prevalence $(4.0 \%)$ was observed in Dole in the West district. Larval counts were usually low; the two highest counts (i.e. 4 and 9 larvae) were observed in stool samples from the two infected children in Dole in the West district.

In Banda-Maji village in North A district, more than two-thirds of the children were infected with at least one helminth species $(69.6 \%)$. The second highest prevalence of helminth infection was observed in Kitumba village located in Central district $(52.0 \%)$. Considerably lower prevalences were found in Mahonda village in North B district $(40.0 \%)$, in Pete village in South district $(39.1 \%)$, in
Dole village in West district $(30.8 \%)$ and, finally, in the primary schools surveyed in the Urban district $(22.4 \%)$. Taken together, almost half of the children surveyed were infected with at least one helminth (166/336, overall prevalence: 49.4\%). Moreover, 47 $(28.3 \%)$ children harboured two or more helminth species concurrently.

T. trichiura was the predominant helminth with an overall prevalence of $35.5 \%$ as assessed by a single Kato-Katz thick smear per child. The infection prevalence ranged from $16.1 \%$ (Urban district) to $55.2 \%$ (North A district). The large majority of infections $(111 / 116,95.7 \%)$ were of light intensity with EPGs below 1,000 (Table 3). The remaining five children $(4.3 \%)$ had a moderate infection intensity and attended the "madrassas" of either Pete ( $\mathrm{n}$ = 3) or Banda-Maji $(\mathrm{n}=2)$. The overall prevalence of A. lumbricoides infection was $12.2 \%$ according to the Kato-Katz method with the highest prevalence $(40.4 \%)$ observed in Banda-Maji in North A district. Most infections (32/40, 80\%) were of light intensity with EPGs below 5,000. The remaining eight children $(20.0 \%)$ had a moderate infection intensity and they were diagnosed either in BandaMaji in North A district $(n=6)$ or in Dole in West district $(n=2)$. The combined results from the KatoKatz and the Koga agar plate methods revealed an overall hookworm prevalence of $11.9 \%$, ranging

Table 2. Prevalence (number of children positive/number of children examined) of soil-transmitted helminth infections in 336 children from the six districts of Zanzibar, Tanzania.

\begin{tabular}{|c|c|c|c|c|c|}
\hline \multirow[t]{2}{*}{ Village } & \multirow[t]{2}{*}{ District } & \multicolumn{4}{|c|}{ Parasite (method) } \\
\hline & & $\begin{array}{l}\text { A. lumbricoides } \\
\left(\mathrm{K}-\mathrm{K}^{\mathrm{a}}\right)\end{array}$ & $\begin{array}{l}\text { T. trichiura } \\
\left(\mathrm{K}-\mathrm{K}^{\mathrm{a}}\right)\end{array}$ & $\begin{array}{l}\text { Hookworm } \\
\left(\mathrm{K}-\mathrm{K}^{\mathrm{a}}, \mathrm{KAP} \mathrm{P}\right)\end{array}$ & $\begin{array}{l}\text { S. stercoralis } \\
\left(\mathrm{KAP}^{\mathrm{b}}, \mathrm{BM}^{\mathrm{c}}\right)\end{array}$ \\
\hline Banda-Maji & North A & $40.3(27 / 67)$ & $55.2(37 / 67)$ & $14.5(10 / 69)$ & $2.9(2 / 68)$ \\
\hline Mahonda & North B & $8.3(2 / 24)$ & $29.2(7 / 24)$ & $16.0(4 / 25)$ & $0(0 / 21)$ \\
\hline Kitumba & Central & $9.1(7 / 77)$ & $44.2(34 / 77)$ & $11.7(9 / 77)$ & $2.8(2 / 74)$ \\
\hline Dole & West & $5.9(3 / 51)$ & $19.6(10 / 51)$ & $15.4(8 / 52)$ & $4.0(2 / 50)$ \\
\hline Pete & South & $2.2(1 / 46)$ & $39.1(18 / 46)$ & $6.5(3 / 46)$ & $0(0 / 39)$ \\
\hline- & Urban & $0(0 / 62)$ & $16.1(10 / 62)$ & $7.7(5 / 65)$ & $1.5(1 / 67)$ \\
\hline Total & & $12.2(40 / 327)$ & $35.5(116 / 327)$ & $11.9(39 / 334)$ & $2.2(7 / 319)$ \\
\hline
\end{tabular}

${ }^{\mathrm{a}} \mathrm{K}-\mathrm{K}=$ Kato-Katz thick smear; ${ }^{\mathrm{b}} \mathrm{KAP}=\mathrm{Koga}$ agar plate; ${ }^{\mathrm{c}} \mathrm{BM}=$ Baermann 
Table 3. Characteristics of the three common soil-transmitted helminth infections among the 327 school-aged children from Unguja who had one stool sample quantitatively examined by the Kato-Katz method.

\begin{tabular}{llll}
\hline Characteristics & \multicolumn{2}{c}{ Parasite } \\
\hline & T. trichiura & A. lumbricoides & Hookworm \\
\cline { 2 - 4 } Number (\%) of children infected & $116(35.5 \%)$ & $40(12.2 \%)$ & $32(9.8 \%)$ \\
Infection intensity & & & $32(80.0 \%)$ \\
Light & $111(95.7 \%)$ & $8(20.0 \%)$ & 0 \\
Moderate & $5(4.3 \%)$ & 0 & 0 \\
Heavy & 0 & $104(86-125)$ & $143(98-207)$ \\
Geometric mean EPG $(95 \% \mathrm{CI})$ & $1,326(962-1,829)$ & & \\
\hline
\end{tabular}

$\mathrm{CI}=$ confidence interval; $\mathrm{EPG}=$ eggs per gram of stool

between $6.5 \%$ (Pete, South district) and 16.0\% (Mahonda, North B district). According to the quantitative Kato-Katz thick smear results, all infections were of light intensity with EPGs below 2,000.

The geometric mean EPGs of the three common soil-transmitted helminths are summarised in Table 3.

\section{Geological features and soil types}

The geographical location of the six sites where the study was carried out were superimposed onto a Google ${ }^{\mathrm{TM}}$-Earth satellite image of Unguja (Fig. 1B) and juxtaposed to the underground composition and predominant soil types of the island (Fig. 1A), according to geological investigations published by Kent and colleagues in the early 1970s (Kent et al., 1971). The geographical coordinates of the study sites are as follows: Banda-Maji ( $5^{\circ} 57^{\prime} 0^{\prime \prime} \mathrm{S}$ longitude and $39^{\circ}$ 19' 0" E latitude); Mahonda (5 59' 0" S, 39 15' 0" E); Kitumba (6 $6^{\circ} 07^{\prime} 0$ " S, $39^{\circ} 17^{\prime}$ 0” E); Dole

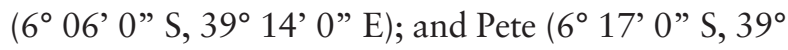
$\left.25^{\prime} 0^{\prime \prime} \mathrm{E}\right)$. The urban area is located in Zanzibar Town (6 $6^{\circ} 09^{\prime} 0$ " S, 39 $39^{\circ}$ ' 0" E).

The ground in Banda-Maji in North A district and in Kitumba in Central district consists of fossiliforous limestone and marly sand lithology. In Mahonda in North B district and in Dole in West district, the ground is characterized by clayey sands with subordinate limestone. In Pete in South district, reefal limestone constitutes the ground. In the urban area around Zanzibar Town, the ground consists primarily of limestone and soft sandstone or alluvium.

\section{Discussion}

Despite considerable efforts to control helminth infections on the islands of Unguja and Pemba over the past decade (Renganathan et al., 1995; Mohammed et al., 2008), surprisingly little is known about the spatial distribution of different helminth species, including underlying demographic, environmental and socio-economic factors. Our study sheds light on the distribution of helminth infections (among children) in the six districts of Unguja, five of them characterized as rural and the sixth in Zanzibar Town being urban. Emphasis is placed on the geological composition and soil types of the study locations, since these environmental factors have been shown to play an important role in the transmission of soiltransmitted helminthiasis (Brooker et al., 2004; Saathoff et al., 2005a). Particular consideration was given to $S$. stercoralis because this helminth species is often neglected in epidemiological investigations, which is partially explained by the need of particular diagnostic methods that are seldom used (Steinmann et al., 2007; Knopp et al., 2008).

In the present study, we screened over 300 children from six environmentally-distinct settings and subjected a single stool sample per child to the Kato- 


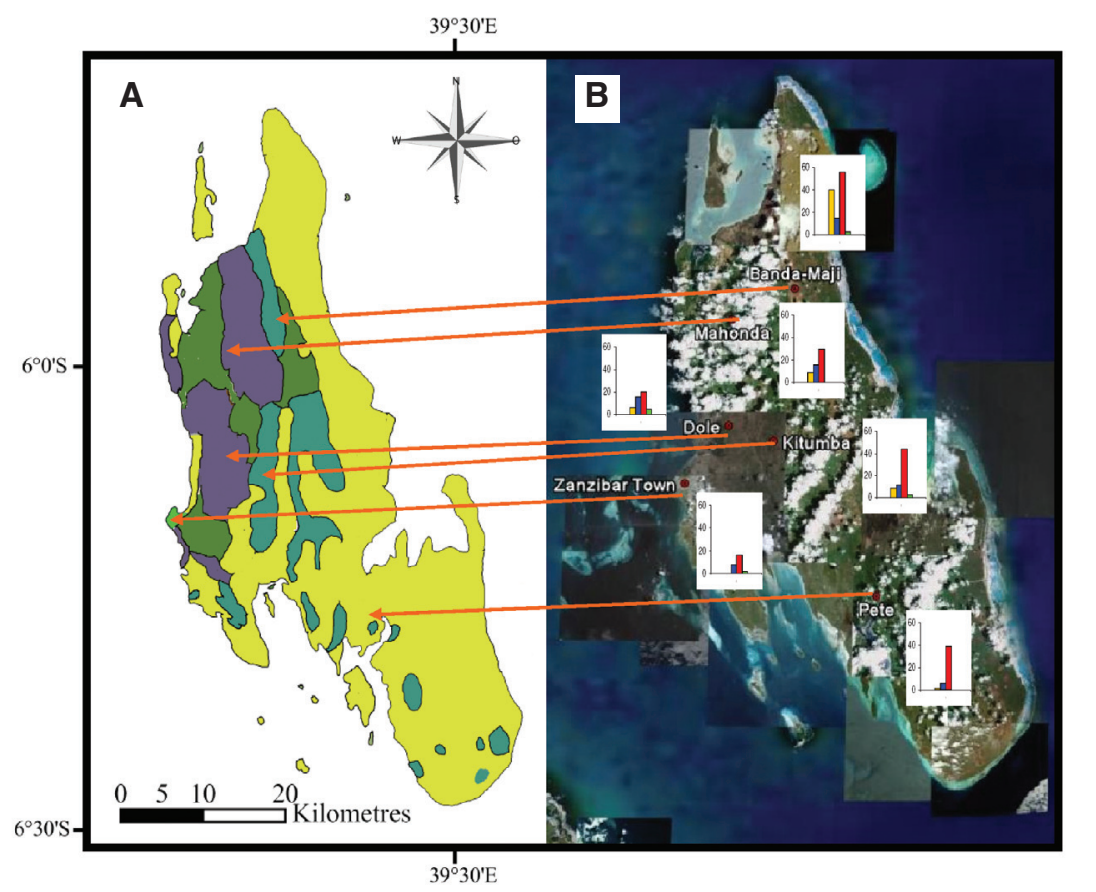

Geological zones (map A)

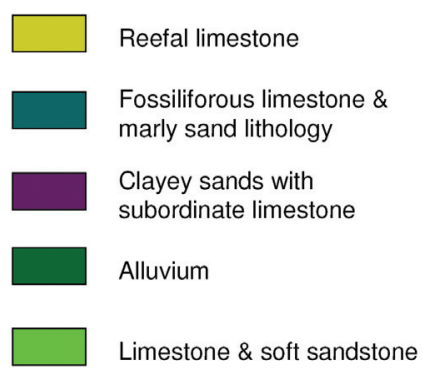

Parasites (map B)

A. lumbricoides

Hookworm

T. trichiura

S. stercoralis

Fig. 1. Map showing the geological zones of Zanzibar (adapted from Kent et al., 1971; A) and satellite image of Zanzibar from Google ${ }^{\mathrm{TM}}$-Earth (B) indicating the surveyed settings.

Katz, Koga agar plate and Baermann methods. We achieved a reasonable compliance; more than $77 \%$ (259/336) of the children supplied stool samples of sufficient quantity to perform all three tests. Our findings underscore the feasibility and efficiency of epidemiological studies carried out in school environments, which in turn further strengthen the collaborative links between the health and education sectors (Lengeler et al., 2002). Approximately half of the children were infected with at least one helminth species with $T$. trichiura being the predominant one. Importantly, helminth infections were primarily of light intensity according to WHO thresholds (Montresor et al., 1998), which show the ability of a rigorously implemented national helminth control programme to reduce morbidity. Whilst $T$. trichiura and hookworm infections were observed in children from all six districts, no A. lumbricoides infections were diagnosed in the urban setting, and S. stercoralis infections were not detected in Mahonda in North B district and Pete in South district. However, the latter findings have to be interpreted with care, as the sample sizes in Mahonda and Pete were small (21 and 39 children, respectively) and only one stool sample per child was examined. Of note, $A$. lumbricoides and hookworm infections were considerably more often diagnosed in the northern part of Unguja than in the South.

We speculate that the high point-prevalence of T. trichiura infections in all districts (i.e. 16-55\%) is, at least partially, explained by the low efficacy of the anthelminthics albendazole and ivermectin against this parasite (Marti et al., 1996; Keiser and Utzinger, 2008). The national control programme, emphasising repeated administration of anthelminthic drugs to school-aged children and other high-risk groups in Unguja, might therefore only have a limited effect on the transmission of trichuriasis. Moreover, it is likely that rapid re-infection occurs as long as sanitation on Unguja remains inadequate, or other treatment regimens (e.g. triple dose) or more efficacious drugs against trichuriasis 
have been developed and used in the national helminth control programme.

Distinct spatial patterns were observed for the other helminth species. From a north-south perspective (not considering the Urban district in the centre), children visiting the "madrassas" in the northern part of the island had a higher risk to be infected with $A$. lumbricoides (odds ratio $(\mathrm{OR})=$ 9.75) and hookworm $(\mathrm{OR}=2.31)$ but a similar risk to be infected with $T$. trichiura $(\mathrm{OR}=1.05)$ compared to the children from the "madrassa" in Pete in South district. However, these findings might be biased by the small sample size in Pete $(\mathrm{n}=39)$ and should hence be interpreted with care. Regarding the individual study-settings, the prevalence of A. lumbricoides in Banda-Maji in North A district $(40.3 \%)$ was 4.4 to 20 -fold higher than in the other districts. This observation confirms that Banda-Maji has an elevated risk for soil-transmitted helminthiasis (Mohammed et al., 2008; Stothard et al., 2008). The high A. lumbricoides prevalence in Banda-Maji gives rise to concerns about whether in this area school-aged children had indeed been treated regularly with anthelminthic drugs and, if so, whether our findings might be an early sign of albendazole resistance development. Interestingly, a recent study carried out in this setting reported a low cure rate (42\%) when A. lumbricoides-infected individuals were given single oral dose of albendazole (Stothard et al., 2008). On the other hand, the fact that no or only very few A. lumbricoides infections were observed in Pete in South district and among children attending peri-urban schools might be explained by the predominant soil types (i.e. limestone and sandstone) in those areas. It is conceivable that these soil types do not provide conducive microenvironments for sustaining the longevity of A. lumbricoides eggs, and that transmission of this infection is hence compromised. In addition, hookworm infections were less prevalent in these two settings. Indeed, the infective larval stages of hookworm require appropriate warmth, humidity and UV protection (e.g. facilitated by vegetation coverage providing sufficient shade and moisture of the soil) for their survival, and hence transmission (Brooker et al., 2004; Saathoff et al., 2005b; Hotez, 2008). Thus, the environment in the Unguja's South district might be less suitable for their development and survival when compared to the districts further north. The predominant geological formation in the southern part of Unguja is reefal limestone with dry and sunlight-exposed savannah, whereas the areas studied north of Zanzibar Town consist of alluvium, marly and clayey sands, have higher annual precipitation and include lots of streams and shady forests, which provide more suitable conditions for hookworm larval development and survival. However, the absence of $A$. lumbricoides infections and the low hookworm prevalences in the Urban district are likely also a result of improved socio-economic status leading to behavioural changes (e.g. wearing shoes) and improved hygienic conditions, and hence a reduced risk of infection. Of note, the stool samples obtained in the Urban district were provided by children attending primary schools, which are regularly subjected to screenings and anthelminthic drug distributions conducted by the national helminth control team. The stool samples obtained from the other districts of Unguja stemmed from children visiting "madrassas" which are not specifically part of the periodic school-based mass-drug administrations as children also visit public schools and anthelminthics are additionally distributed to the whole eligible island population in the frame of the programme to eliminate lymphatic filariasis. However, confounding factors such as lower coverage of treatment in the respective study population cannot be excluded.

The highest prevalence of $S$. stercoralis was found in Dole in West district $(4.0 \%)$, but infections were absent in Mahonda in North B district and Pete in South district. Bearing in mind that the absence of an infection might be explained by the low sample sizes there, an additional factor to note is that, in Pete, the environment is drier than elsewhere, which might 
therefore be less suitable for the development of infective $S$. stercoralis larvae or free-living adult stages. However, one has to keep in mind that autoinfection can perpetuate $S$. stercoralis transmission for an extended period of time (Keiser and Nutman, 2004; Vadlamudi et al., 2006). It follows that this helminth is less closely linked to environmental factors than, for example, hookworm larvae. Autoinfection of S. stercoralis also imposes a considerable problem on copro-diagnostics. As larvae are not necessarily excreted in the stool, it is exceedingly difficult to identify infected individuals. Our recent work, focussing on the diagnosis of $S$. stercoralis among schoolchildren in Unguja, showed that the observed prevalence of this worm was more than double when three instead of a single stool sample were examined with a combination of the Koga agar plate and Baermann method (Knopp et al., 2008). Hence, the "true" overall prevalence of $S$. stercoralis in the present study might well be $4 \%$ or even higher.

We conclude that soil-transmitted helminth infections are still prevalent across Unguja, but infection intensities are generally low, demonstrating the positive effect of the national control programme on morbidity. T. trichiura infections seem to be hardest to reduce with the current single-dose anthelminthic treatment campaigns. Although the observed prevalence of $S$. stercoralis was low, the "true" prevalence might be considerably higher were more sensitive diagnostic approaches employed, and hence this parasite should not be neglected. Our findings call for continuation of the national control programme especially in the rural areas, but chemotherapy should be complemented by improved access to clean water and adequate sanitation, coupled with sound health education.

\section{Acknowledgements}

We thank all the children and their parents and/or legal guardians for their collaboration, and we are grateful to the shehas, headmasters and teachers for their support. Thanks are addressed to the staff of the HCLU (Ministry of Health and Social Welfare), especially Alisa Mohd, Haji Ameri and
Alippo Naim for expert help in the field and at the bench. This investigation received financial support from the Swiss National Science Foundation (project no. PPOOB-102883 and PPOOB-119129), the Natural History Museum, London (The Health Foundation, UK), the World Health Organization (OD/TS-07-00331), and the European Union (FP6 STREP CONTRAST project, contract no. 032203).

\section{References}

Albonico M, Chwaya HM, Montresor A, Stolfzfus RJ, Tielsch JM, Alawi KS, Savioli L, 1997. Parasitic infections in Pemba Island school children. East Afr Med J 74, 294-298.

Bethony J, Brooker S, Albonico M, Geiger SM, Loukas A, Diemert D, Hotez PJ, 2006. Soil-transmitted helminth infections: ascariasis, trichuriasis, and hookworm. Lancet 367, 1521-1532.

Brooker S, Bethony J, Hotez PJ, 2004. Human hookworm infection in the 21st century. Adv Parasitol 58, 197-288.

Calton WE, Tidbury GE, Walker GF, 1955. A study of the more important soils of the Zanzibar protectorate. East Afr Agricult J 21, 53-60.

French MD, Rollinson D, Basáñez MG, Mgeni AF, Khamis IS, Stothard JR, 2007. School-based control of urinary schistosomiasis on Zanzibar, Tanzania: monitoring microhaematuria with reagent strips as a rapid urological assessment. J Pediatr Urol 3, 364-368.

Gann PH, Neva FA, Gam AA, 1994. A randomized trial of single- and two-dose ivermectin versus thiabendazole for treatment of strongyloidiasis. J Infect Dis 169, 1076-1079.

García LS, Bruckner DA, 2001. Diagnostic medical parasitology. American Society for Microbiology, Washington, DC, USA, pp. 1-791.

Hettige PML, 1990. Evaluation of land resources in Zanzibar Phase I and Phase II. Part I main volume, land evaluation and land sustainability classification - Unguja and Pemba Islands. Food and Agricultural Organization (FAO), Rome, Italy.

Hotez P, 2008. Hookworm and poverty. Ann N Y Acad Sci 1136, 38-44.

Katz N, Chaves A, Pellegrino J, 1972. A simple device for quantitative stool thick-smear technique in schistosomiasis mansoni. Rev Inst Med Trop São Paulo 14, 397-400.

Keiser PB, Nutman TB, 2004. Strongyloides stercoralis in the immunocompromised population. Clin Microbiol Rev 17, 


\section{8-217.}

Keiser J, Utzinger J, 2008. Efficacy of current drugs against soil-transmitted helminth infections: systematic review and meta-analysis. JAMA 299, 1937-1948.

Kent PE, Hunt JA, Johnstone DW, 1971. The geology and geophysics of coastal Tanzania. Geophysical Paper 6, 100.

Knopp S, Mgeni AF, Khamis IS, Steinmann P, Stothard JR, Rollinson D, Marti HP, Utzinger J, 2008. Diagnosis of soiltransmitted helminths in the era of preventive chemotherapy: effect of multiple stool sampling and use of different diagnostic techniques. PLoS Negl Trop Dis 2, e331.

Koga K, Kasuya S, Khamboonruang C, Sukavat K, Nakamura Y, Tani S, Ieda M, Tomita K, Tomita S, Hattan N, Mori M, Makino S, 1990. An evaluation of the agar plate method for the detection of Strongyloides stercoralis in northern Thailand. J Trop Med Hyg 93, 183-188.

Lengeler C, Utzinger J, Tanner M, 2002. Questionnaires for rapid screening of schistosomiasis in sub-Saharan Africa. Bull World Health Organ 80, 235-242.

Marti HP, Haji HJ, Savioli L, Chwaya HM, Mgeni AF, Ameir JS, Hatz C, 1996. A comparative trial of a single-dose ivermectin versus three days of albendazole for treatment of Strongyloides stercoralis and other soil-transmitted helminth infections in children. Am J Trop Med Hyg 55, 477-481.

MDG-Centre, 2007. An assessment of rainwater harvesting potential in Zanzibar.

Mohammed KA, Haji HJ, Gabrielli A, Mubila L, Biswas G, Chitsulo L, Bradley MH, Engels D, Savioli L, Molyneux $\mathrm{DH}, 2008$. Triple co-administration of ivermectin, albendazole and praziquantel in Zanzibar: a safety study. PLoS Negl Trop Dis 2, e171.

Mohammed KA, Molyneux DH, Albonico M, Rio F, 2006. Progress towards eliminating lymphatic filariasis in Zanzibar: a model programme. Trends Parasitol 22, 340-344.

Monkul MM, Ozden G, 2007. Compressional behavior of clayey sand and transition fines content. Eng Geol 89, 195-205.

Montresor A, Crompton DWT, Hall A, Bundy DAP, Savioli L, 1998. Guidelines for the evaluation of soil-transmitted helminthiasis and schistosomiasis at community level. A guide for managers of control programmes. World Health Organization (WHO), Geneva, Switzerland, pp. 1-45.

Renganathan E, Ercole E, Albonico M, De Gregorio G, Alawi
KS, Kisumku UM, Savioli L, 1995. Evolution of operational research studies and development of a national control strategy against intestinal helminths in Pemba island, 198892. Bull World Health Organ 73, 183-190.

Rudge JW, Stothard JR, Basáñez MG, Mgeni AF, Khamis IS, Khamis AN, Rollinson D, 2008. Micro-epidemiology of urinary schistosomiasis in Zanzibar: local risk factors associated with distribution of infections among schoolchildren and relevance for control. Acta Trop 105, 45-54.

Saathoff E, Olsen A, Kvalsvig JD, Appleton CC, Sharp B, Kleinschmidt I, 2005a. Ecological covariates of Ascaris lumbricoides infection in schoolchildren from rural KwaZulu-Natal, South Africa. Trop Med Int Health 10, 412-422.

Saathoff E, Olsen A, Sharp B, Kvalsvig JD, Appleton CC, Kleinschmidt I, 2005b. Ecologic covariates of hookworm infection and reinfection in rural Kwazulu-natal/South Africa: a geographic information system-based study. Am J Trop Med Hyg 72, 384-391.

Steinmann P, Zhou XN, Du ZW, Jiang JY, Wang LB, Wang XZ, Li LH, Marti H, Utzinger J, 2007. Occurrence of Strongyloides stercoralis in Yunnan province, China, and comparison of diagnostic methods. PLoS Negl Trop Dis 1, e75.

Stoltzfus RJ, Albonico M, Tielsch JM, Chwaya HM, Savioli L, 1997. School-based deworming program yields small improvement in growth of Zanzibari school children after one year. J Nutr 127, 2187-2193.

Stothard JR, Imison E, French MD, Sousa-Figueiredo JC, Khamis IS, Rollinson D, 2008. Soil-transmitted helminthiasis among mothers and their pre-school children on Unguja Island, Zanzibar with emphasis upon ascariasis. Parasitology (in press).

Stothard JR, Mgeni AF, Khamis S, Seto E, Ramsan M, Hubbard SJ, Kristensen TK, Rollinson D, 2002. New insights into the transmission biology of urinary schistosomiasis in Zanzibar. Trans R Soc Trop Med Hyg 96, 470-475. Stothard JR, Rollinson D, 1997. Molecular characterization of Bulinus globosus and B. nasutus on Zanzibar, and an investigation of their roles in the epidemiology of Schistosoma haematobium. Trans R Soc Trop Med Hyg 91, 353-357.

Vadlamudi RS, Chi DS, Krishnaswamy G, 2006. Intestinal strongyloidiasis and hyperinfection syndrome. Clin Mol Allergy 4, 8 . 